\title{
Quantum interference of photon pairs from two remote trapped atomic ions
}

\author{
P. MAUNZ*, D. L. MOEHRING, S. OLMSCHENK, K. C. YOUNGE, D. N. MATSUKEVICH AND C. MONROE \\ FOCUS Center and Department of Physics, University of Michigan, Ann Arbor, Michigan 48109-1040, USA \\ *e-mail: pmaunz@umich.edu
}

Trapped atomic ions are among the most attractive implementations of quantum bits for applications in quantuminformation processing, owing to their long trapping lifetimes and long coherence times. Although nearby trapped ions can be entangled through their Coulomb-coupled motion ${ }^{1-6}$, it seems more natural to entangle remotely located ions through a coupling mediated by photons, eliminating the need to control the ion motion. A promising way to entangle ions via a photonic channel is to interfere two photons emitted from the ions and then detect appropriate photon coincidence events ${ }^{7-9}$. Here, we report the pivotal element of this scheme in the observation of quantum interference between pairs of single photons emitted from two atomic ions residing in independent traps.

Remote entanglement of two ions or atoms can be achieved by subjecting two photons emitted by the particles to a Bellstate measurement and is heralded by an appropriate coincidence detection of the photons ${ }^{7}$. The essence of this Bell-state measurement is the quantum interference of two photons, which has been observed previously with photons generated in a variety of physical processes and systems, including nonlinear optical down-conversion ${ }^{10,11}$, quantum dots ${ }^{12}$, atoms in cavity quantum electrodynamics ${ }^{13}$, atomic ensembles ${ }^{14-16}$ and two nearby trapped neutral atoms ${ }^{17}$. We report the first observation of interference between two single photons emitted from two remote trapped atomic ions. The two $\mathrm{Yb}$ ions are stored in independent traps in two vacuum chambers separated by about one metre. The interference of two single photons emitted by remote ions is the only element of remote-entanglement schemes that has not previously been demonstrated. Hence, this demonstration is an essential step towards future remote-ion-entanglement experiments, which may ultimately lead to large-scale quantum networks ${ }^{18-23}$.

In the experiment, single ${ }^{174} \mathrm{Yb}^{+}$ions are trapped in two congeneric Paul traps located in separate vacuum chambers as described in detail in the Methods section. Laser cooling localizes the ions to within the resolution of the diffraction-limited imaging optics but well outside the Lamb-Dicke limit. The ions are excited with ultrafast laser pulses generated by a picosecond mode-locked Ti:sapphire laser with a centre frequency of $739 \mathrm{~nm}$. Each pulse is then frequency doubled to $369.5 \mathrm{~nm}$ through a phase-matched lithium triborate nonlinear crystal. An electro-optic pulse picker is used to reduce the pulse repetition rate from $81 \mathrm{MHz}$ to $8.1 \mathrm{MHz}$ with an extinction ratio of better than $10^{4}: 1$. The second harmonic is filtered from the fundamental with a prism, split between the two traps using a beam splitter and aligned to arrive at the two ions within 100 ps of each other. Each pulse has a neartransform-limited pulse duration of 2 ps and excites the ions on a timescale much faster than the excited-state lifetime of $8 \mathrm{~ns}$. The
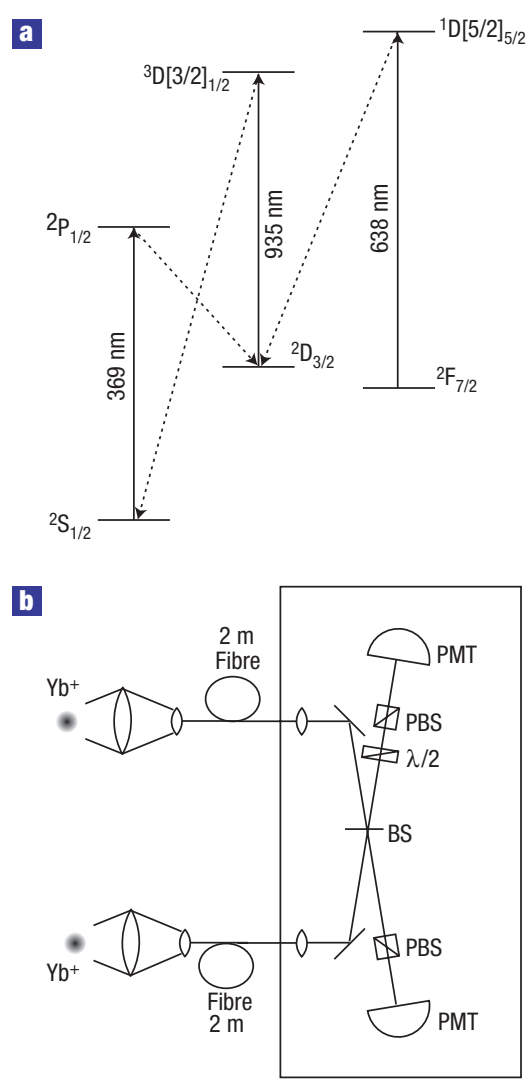

Figure 1 The ytterbium set-up. a, Relevant level scheme of ${ }^{174} \mathrm{Yb}^{+}$. b, Schematic diagram of the detection system. The unpolarized light from each ${ }^{174} \mathrm{Yb}^{+}$ion is collected using a triplet lens with a numerical aperture of 0.23 and a working distance of $13 \mathrm{~mm}$. It is coupled into a single-mode fused-silica fibre using an aspheric collimation lens with a numerical aperture of 0.6 . About $25 \%$ of the light collected from each ion is transmitted through the $2 \mathrm{~m}$ fibres. The light from both fibres is collimated with a monochromatic lens and mode matched on a polarization-independent beam splitter (BS). Behind the beam splitter, two removable polarizers (PBS) can be used to select parallel polarization. Inserting a $\lambda / 2$ plate allows the detection of perpendicularly polarized photons. Subsequently, the light is detected by two photon-counting photomultipliers (PMT) with a quantum efficiency of about $20 \%$ and a time resolution of about $1 \mathrm{~ns}$. The photon arrival times at the photomultipliers are recorded using a time-to-digital converter with a resolution of $4 \mathrm{ps}$ (PicoQuant PicoHarp300). The contrast of the interferometer is measured using $369.5 \mathrm{~nm}$ laser light to be better than $96 \%$. 

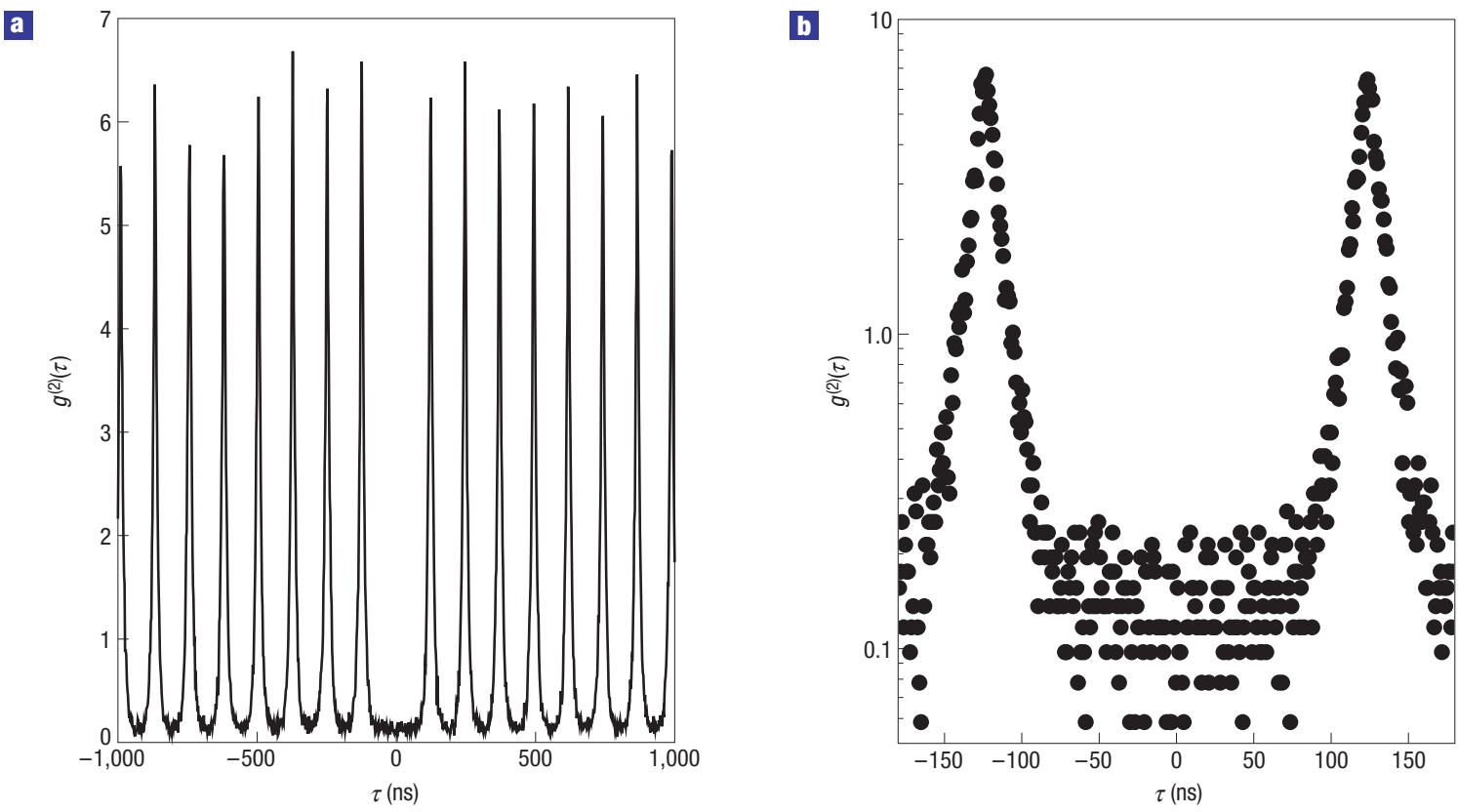

Figure 2 Intensity autocorrelation of the light emitted by a single ion excited by picosecond pulses. a, The periodically emitted photons from the pulsed excitation lead to peaks at multiples of the $124 \mathrm{~ns}$ pulse repetition time. $\mathbf{b}$, The near-perfect photon antibunching, revealed by the logarithmic scale, proves that at most one photon is emitted from an excitation pulse. The correlation function is evaluated from the stored photon arrival times using $2 \mathrm{~ns}$ binning. The probability to detect a photon from one excitation pulse is $6 \times 10^{-4}$. The data shown were integrated for $3 \mathrm{~h}$.

pulse energy can be adjusted to simultaneously excite both ions with near-unit probability.

Two re-entrant viewports at opposing sides of each trap are used to simultaneously image the ions in free space and couple light scattered from the ions into single-mode optical fibres. In free space, a telescope is used to image scattered photons on a camera to monitor loading and to verify the presence of a single ${ }^{174} \mathrm{Yb}^{+}$ion in each trap. Fibre coupling is realized from the opposite side of the trap using a second objective lens to collect spontaneously emitted photons (Fig. 1b). Each beam is focused onto a single-mode fibre using an aspheric lens. About $25 \%$ of the spontaneously emitted photons collected by the objective are transmitted through the $2 \mathrm{~m}$ fibre. The ion light transmitted through the single-mode fibres is collimated and mode matched on a polarization-independent beam splitter. The light emerging from the beam splitter is detected using photomultipliers. Removable polarizers and a removable $\lambda / 2$ wave plate allow detection of unpolarized photons, or photons of parallel or perpendicular polarization. The arrival times of the photons at the photomultipliers are recorded using a time-todigital converter.

To demonstrate that the excitation of an ion with an ultrashort pulse leads to the emission of at most one photon, we first analyse the light scattered by a single ${ }^{174} \mathrm{Yb}^{+}$ion by blocking the fluorescence from one of the two traps. We use a repetitive sequence consisting of a $40 \mu$ s measurement interval and a $10 \mu$ s cooling interval. During the cooling interval, the ion is Doppler cooled only with continuous-wave (cw) light, whereas during the measurement interval, the ion is excited only by ultrafast laser pulses with a $124 \mathrm{~ns}$ pulse separation. From the arrival times of the photons at the photomultipliers, the intensity autocorrelation function, $g^{(2)}(\tau)$, is evaluated and shown in Fig. 2. Here, $\tau$ is the detection-time delay of two photons at the two detectors. The periodic ultrafast excitation of the single ion leads to peaks at multiples of the pulse separation time. As the ion is excited on a timescale much faster than the excited-state lifetime, the spontaneously emitted photons have an exponential envelope ${ }^{24}$. Therefore, the peaks in the autocorrelation function have a two-sided exponential decay where the $1 / \mathrm{e}$ half width is given by the $8 \mathrm{~ns}$ lifetime of the excited state. In contrast to pulsed coherent or pulsed thermal light, there is no peak at zero time delay (Fig. 2). This near-perfect antibunching is highly non-classical and clearly demonstrates that at most one photon is emitted after each excitation pulse. Theoretically, the probability to scatter two photons from one ion excited with one pulse is limited by the emission probability of an excited atom during the excitation pulse $\left(\approx 10^{-4}\right.$ here). This very clean single-photon source is a good starting point for the observation of two-photon interference and is necessary for high-fidelity photon-mediated ion entanglement.

Two-photon interference is a purely quantum phenomenon and can be understood qualitatively by considering the four possibilities in which two photons impinging on different input ports of a beam splitter can emerge from the output ports. In two of these possibilities, the two photons leave from different ports and thus can generate a coincidence event: both photons are reflected, or both are transmitted. For two photons that have the same polarization, frequency and are spatially mode matched on the beam splitter, these two cases interfere destructively. This leads to the effect that the photons always emerge together from the beam splitter and no coincidence events are observed ${ }^{25}$. This can also be understood from the bosonic nature of the two-photon system ${ }^{9}$.

To observe two-photon interference, the single ions in both traps are excited simultaneously by picosecond laser pulses and the fluorescence photons are combined on the beam splitter. The polarizers after the beam splitter are used to measure the coincidences of parallel polarized photons. To measure coincidences of perpendicularly polarized photons in the two output ports of the beam splitter, a $\lambda / 2$ wave plate is added in front of one polarizer. The resulting cross-correlation functions are shown in Fig. 3. For perpendicular polarization, the photons 


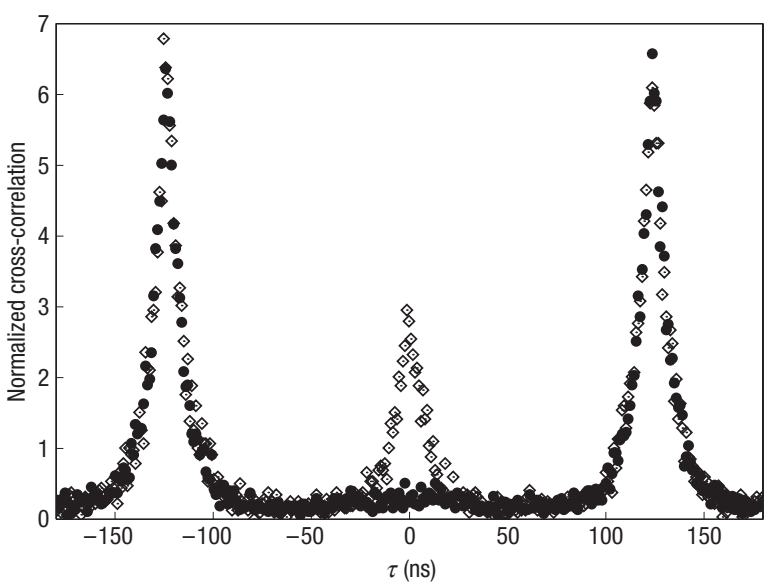

Figure 3 Normalized intensity cross-correlation of photons emitted by two ions. For perpendicular linear polarization (diamonds) the photons from the two ions are distinguishable; thus we observe a peak at zero delay. In contrast to the peaks at longer delay times, for which the two photons can be emitted by the same ion, the two photons leading to a coincidence at zero delay must be emitted by different ions. Thus, the peak at zero delay has half the area of the peaks at longer delay times. Integrating all photon pairs with a detection-time difference of $|\tau|<8 \mathrm{~ns}$ results in a coincidence detection probability of $1.4 \times 10^{-8}$ per excitation pulse (or $2 \times 10^{-8}$ for the photon pairs with a less than 16 ns detection-time difference). For parallel linear polarization (circles), the two-photon interference suppresses the coincidence detection of two photons on different output ports of the beam splitter. Including dark counts, the number of photon pairs with less than $8 \mathrm{~ns}$ and $16 \mathrm{~ns}$ detection-time differences is reduced by $86 \%$ and $81 \%$, respectively. Each curve was integrated for about $4 \mathrm{~h}$ and evaluated using a $1 \mathrm{~ns}$ binning.

are distinguishable and do not interfere; thus, we find a peak at zero delay. This peak has half the area of the others, because at zero delay each ion can only contribute one photon, whereas for non-simultaneous pulses each ion can produce both photons in the correlation measurement. Integrated over photon pairs detected within $8 \mathrm{~ns}$ of each other (one lifetime), this peak consists of 1,087 coincidences corresponding to a coincidence detection probability of $1.4 \times 10^{-8}$ per excitation pulse or an absolute coincidence rate of about $0.1 \mathrm{~s}^{-1}$. For parallel polarization of the two photons, the two-photon interference eliminates coincidence detections. In our case, the remaining peak at zero delay, after subtracting the dark-count background, has an amplitude of about $5 \%$ of the perpendicularly polarized signal, corresponding to a $95 \%$ contrast of the interferometer. Integrating all photon pairs that are detected within $8 \mathrm{~ns}$ of each other, including dark counts, we detect $14 \%$ of the photon pairs that we observe for perpendicular polarization.

To use this two-photon interference to produce remote-ion entanglement, an attribute of each photon-polarization ${ }^{26}$ or frequency ${ }^{23}$ - must be entangled with the internal state of its parent ion. A coincidence detection of two photons would then project the system onto the antisymmetric superposition state and herald the entanglement of the ions ${ }^{7-9}$. The interference contrast observed here, including dark counts, would lead to a potential entanglement fidelity of about $90 \%$. The fidelity is limited by the ratio between photon detection events and dark counts. This ratio can be improved by lowering detector dark counts, achieving a higher detection efficiency or by collecting photons from a larger effective solid angle. The most promising way to implement this entanglement scheme with distant ytterbium ions uses the ground-state hyperfine clock states of ${ }^{171} \mathrm{Yb}^{+}$to encode the atomic qubit. To realize this experimentally, additional laser frequencies are necessary for cooling of ${ }^{171} \mathrm{Yb}^{+}$. In addition, efficient state initialization and state detection in any qubit basis must be implemented to generate and verify the entanglement ${ }^{27}$.

In summary, we demonstrate a near-perfect single-photon source on the basis of the ultrafast excitation of a single trapped ion and two-photon quantum interference of photon pairs emitted by two remote trapped ions. The contrast of the observed interference in principle allows the entanglement of ions with a fidelity of about $90 \%$, clearly surpassing the threshold needed to violate a Bell inequality. Building on this, entangling gates could provide a means to scale the probabilistic entanglement from two qubits to the generation of networks of entangled qubits and cluster states. The tremendous advantages of the trapped-ion system-extremely long storage and coherence times and high readout fidelity—should make the scalable entanglement of many qubits feasible.

\section{METHODS}

The experimental apparatus consists of two congeneric radiofrequency (rf) Paul traps located in separate vacuum chambers. These four-rod linear quadrupole traps have rod spacings of $0.5 \mathrm{~mm}$ and end-cap spacings of $2.6 \mathrm{~mm}$. The $\mathrm{rf}$ drive frequency is $\Omega_{\mathrm{T}} / 2 \pi \approx 37 \mathrm{MHz}$ and the centre-of-mass secular trapping frequencies are $\left(\omega_{x}, \omega_{y}, \omega_{z}\right) / 2 \pi \approx(1,1,0.2) \mathrm{MHz}$. Residual micromotion at the $\mathrm{rf}$ drive frequency is carefully reduced by applying static offset voltages to the trap rods. $\mathrm{Yb}$ atoms from a thermal beam are photoionized by resonantly exciting the ${ }^{1} \mathrm{~S}_{0} \leftrightarrow{ }^{1} \mathrm{P}_{1}$ transition near $399 \mathrm{~nm}$ using a diode laser and subsequently promoting the electron to the continuum with a $369.5 \mathrm{~nm} \mathrm{cw}$ laser $^{27}$, which is also used to Doppler cool the ions. This $369.5 \mathrm{~nm}$ laser light is generated by frequency doubling the light from a cw amplified diode laser at $739 \mathrm{~nm}$ and is stabilized to an iodine reference at a detuning of $-10 \mathrm{MHz}$ from the atomic ${ }^{2} \mathrm{~S}_{1 / 2} \leftrightarrow{ }^{2} \mathrm{P}_{1 / 2}$ transition of ${ }^{174} \mathrm{Yb}^{+}$(linewidth $\approx 20 \mathrm{MHz}$ ) (Fig. 1a). The ions reside in a magnetic field of about $10 \mathrm{G}$ to prevent pumping into dark states. From the excited state, the ion can decay to the ${ }^{2} \mathrm{D}_{3 / 2}$ state with a probability of 0.05 (ref. 28). A diode laser near $935 \mathrm{~nm}$ is used to repump the ion via the ${ }^{3} \mathrm{D}[3 / 2]_{1 / 2}$ state to the ground state. About once an hour the ion reaches the ${ }^{2} \mathrm{~F}_{7 / 2}$ state, which is cleared by a diode laser near $638 \mathrm{~nm}$. The mean storage time of the ions in the trap is several days. All of the data presented here were obtained with the same two ions.

Received 2 March 2007; accepted 17 May 2007; published 10 June 2007.

References

1. Cirac, J. I. \& Zoller, P. Quantum computations with cold trapped ions. Phys. Rev. Lett. 74, 4091-4094 (1995).

2. Mølmer, K. \& Sørensen, A. Multiparticle entanglement of hot trapped ions. Phys. Rev. Lett. 82, 1835-1838 (1999).

3. Milburn, G. J., Schneider, S. \& James, D. F. V. Ion trap quantum computing with warm ions. Fortschr. Phys. 48, 801-810 (2000).

4. Sackett, C. A. et al. Experimental entanglement of four particles. Nature 404, 256-259 (2000).

5. Leibfried, D. et al. Creation of a six-atom 'Schrödinger cat' state. Nature 438, 639-642 (2005).

6. Häffner, H. et al. Scalable multiparticle entanglement of trapped ions. Nature 438, 643-646 (2005).

7. Simon, C. \& Irvine, W. T. M. Robust long-distance entanglement and a loophole-free Bell test with ions and photons. Phys. Rev. Lett. 91, 110405 (2003).

8. Duan, L. M., Blinov, B. B., Moehring, D. L. \& Monroe, C. Scalable trapped ion quantum computation with a probabilistic ion-photon mapping. Quant. Inf. Comp. 4, 165-173 (2004).

9. Moehring, D. L. et al. Quantum networking with photons and trapped atoms. J. Opt. Soc. Am. B 24 300-315 (2007).

10. Hong, C. K., Ou, Z. Y. \& Mandel, L. Measurement of subpicosecond time intervals between two photons by interference. Phys. Rev. Lett. 59, 2044-2046 (1987).

11. Kaltenbaek, R., Blauensteiner, B., Żukowski, M., Aspelmeyer, M. \& Zeilinger, A. Experimental interference of independent photons. Phys. Rev. Lett. 96, 240502 (2006).

12. Santori, C., Fattal, D., Vučković, J., Solomon, G. S. \& Yamamoto, Y. Indistinguishable photons from a single-photon device. Nature 419, 594-597 (2002).

13. Legero, T., Wilk, T., Hennrich, M., Rempe, G. \& Kuhn, A. Quantum beat of two single photons. Phys. Rev. Lett. 93, 070503 (2004).

14. Thompson, J. K., Simon, J., Loh, H. \& Vuletic, V. A high-brightness source of narrowband, identical-photon pairs. Science 313, 74-77 (2006).

15. Felinto, D. et al. Conditional control of the quantum states of remote atomic memories for quantum networking. Nature Phys. 2, 844-848 (2006).

16. Chanelière, T. et al. Quantum interference of electromagnetic fields from remote quantum memories. Phys. Rev. Lett. 98, 113602 (2007).

17. Beugnon, J. et al. Quantum interference between two single photons emitted by independently trapped atoms. Nature 440, 779-782 (2006)

18. Briegel, H. J., Dür, W., Cirac, J. I. \& Zoller, P. Quantum repeaters: The role of imperfect local operations in quantum communication. Phys. Rev. Lett. 81, 5932-5935 (1998).

19. Raussendorf, R. \& Briegel, H. J. A one-way quantum computer. Phys. Rev. Lett. 86, 5188-5191 (2001)

20. Barrett, S. D. \& Kok, P. Efficient high-fidelity quantum computation using matter qubits and linear optics. Phys. Rev. A 71, 060310 (2005). 
21. Duan, L. M. \& Raussendorf, R. Efficient quantum computation with probabilistic quantum gates. Phys. Rev. Lett. 95, 080503 (2005).

22. Lim, Y. L., Beige, A. \& Kwek, L. C. Repeat-until-success linear optics distributed quantum computing. Phys. Rev. Lett. 95, 030505 (2005).

23. Duan, L. M. et al. Probabilistic quantum gates between remote atoms through interference of optical frequency qubits. Phys. Rev. A 73, 062324 (2006).

24. Moehring, D. L. et al. Precision lifetime measurements of a single trapped ion with ultrafast laser pulses. Phys. Rev. A 73, 023413 (2006).

25. Mandel, L. Quantum effects in one-photon and two-photon interference. Rev. Mod. Phys. 71, S274-S282 (1999).

26. Blinov, B. B., Moehring, D. L., Duan, L. M. \& Monroe, C. Observation of entanglement between a single trapped atom and a single photon. Nature 428, 153-157 (2004).

27. Balzer, C. et al. Electrodynamically trapped $\mathrm{Yb}^{+}$ions for quantum information processing. Phys. Rev. A 73, 041407 (2006)
28. Yu, N. \& Maleki, L. Lifetime measurements of the $4 \mathrm{f}^{\mathrm{f}}{ }^{14} \mathrm{~d}$ metastable states in single ytterbium ions. Phys. Rev. A 61, 022507 (2000).

\section{Acknowledgements}

We acknowledge discussions with L.-M. Duan. This work is supported by the National Security Agency and the Disruptive Technology Office under Army Research Office contract

W911NF-04-1-0234, and the National Science Foundation Information Technology Research (ITR) and Physics at the Information Frontier (PIF) programs.

Correspondence and requests for materials should be addressed to P.M

Competing financial interests

The authors declare no competing financial interests.

Reprints and permission information is available online at http://npg.nature.com/reprintsandpermissions/ 